\title{
Broadband characterisation of in-duct acoustic sources using an equivalent source approach
}

\author{
J.W.R.Meggitt ${ }^{1}$, A.S.Elliott ${ }^{1}$, A.T.Moorhouse ${ }^{1}$, G.Banwell ${ }^{2}$, H.Hopper ${ }^{2}$, J.Lamb ${ }^{2}$ \\ ${ }^{1}$ Acoustics Research Centre, University of Salford, Greater Manchester, M5 4WT \\ ${ }^{2}$ Dyson LTD, Malmesbury, SN16 ORP
}

\begin{abstract}
This paper is concerned with the development of an experimental method capable of independently characterising low Mach number, in-duct fluid machines, such as pumps, fans, etc. The aim is to propose and test a source characterisation method that is suitable for acoustic simulation and the construction Virtual Acoustic Prototypes. Such a requirement demands that the source characterisation be (a) independent, so that components can be virtually (re)combined within different assemblies and (b) valid over a wide frequency range so as to enable the output of a virtual assembly to be auralised. In this regard, standard methods based on sound power are not suitable. An equivalent source approach is proposed, based on a two-stage measurement procedure in which source strengths are obtained by solving an inverse problem. The experimental application of the procedure is illustrated as part of a case study where a high speed compressor unit is independently characterised and the resulting source data used to predict the operational response in a new assembly. Three frequency ranges are identified based on plane wave, modal and statistical behaviour within the duct. The same measured data is used within each regime but subject to different processing according to different assumptions. Predictions are shown to be in good agreement with the directly measured response over a broad frequency range $(100 \mathrm{~Hz}$ to $10 \mathrm{kHz})$.
\end{abstract}

Keywords: in-duct, acoustic, source characterisation, inverse, volume velocity

\section{Introduction}

There is currently significant interest in the acoustic simulation of built up assemblies, where components are virtually combined so as to create Virtual Acoustic Prototypes for example of vehicles, buildings and domestic products [1]. This paper addresses the need to characterise in-duct fluid machines as important acoustic sources within this context. To be suitable for use within a Virtual Acoustic Prototype, a source characterisation must (a) provide independent source data, so as to allow components to be (re)combined virtually in different combinations and (b) be valid over a sufficiently wide frequency range for auralisation purposes. Further to providing input to acoustic simulation, an appropriate source characterisations can provide a powerful tool for diagnostics and acoustic design. Hence, the aim of the work presented here is to propose and test a source characterisation method for in-duct fluid machines that satisfies both the requirement of independence and broadband application.

The most standard approach for the characterisation of in-duct sources is based on sound power [2] but is limited to the plane wave region. Additionally, the procedure discards the phase and time structure information important for auralisation. Similar limitations are found with other sound power based methods [3, 4].

In the field of hydraulic fluid power there has been much interest in the measurement and rating of fluid-borne noise emanating from positive displacement pumps. The flow ripple, that is, the fluctuation superimposed over a mean flow rate, is generally considered to be an independent characteristic of the pump, and thus suitable as a fluid-borne noise rating. Its measurement, based on the well established secondary source method [5], has since been standardised [6]. The procedure returns a complex source quantity, thus retaining phase information, but like [2], it is limited to the plane wave region which is generally not sufficient for auralisation.

A more advanced procedure considers the characterisation of an in-duct acoustic source in the modal domain through the application of multi-port theory $[7,8,9,10,11]$. This approach relies on the modal decomposition of a 
wave field, and leads to a definition of the source in terms of outgoing modal wave amplitudes. Whilst phase is retained and higher order modal information accounted for, the multi-port approach requires substantial experimental set-up, making its implementation time consuming and expensive. This is especially so if a characterisation is required up to a high frequency. The multi-port approach has evolved through decades of advancements and the reader is referred to the above references for a more comprehensive accounting of the associated literature.

Another, less invasive, method was investigated in [12], in which Weidmann's acoustic similarity law was used to determine a source's (planar) characteristics by isolating the acoustic loading (acoustic loading is the acoustic impedance of the circuit as seen by the fan as an acoustic source, and is not to be confused with aerodynamic load, which is the pressure drop of the circuit that determines the fan duty, i.e. the operating point of the fan. In this work acoustic load is assumed to be independent of the flow speed.) of the test rig. However, this interesting approach does not appear to have been widely adopted, perhaps because of the demanding measurements it requires and in any case, it would not provide data suitable for auralisation.

In the following, we propose an alternative approach based on the use of an equivalent acoustic source. The proposed method leads to a definition of the source in terms of an acoustic 'free velocity', which is approximated by a number of elementary sources (i.e monopoles). This is not unlike the secondary source method presented in [5, 6]. In the field of structural dynamics a similar equivalent source approach has been used to independently characterise vibration sources in terms of their blocked force [13]. A general theoretical support of the above can be found in [14] where an equivalent field theorem is presented. A special case of this theorem and its main result is provided in Appendix A of this work.

To make an operational prediction in some new installation (i.e. a source-receiver system), the passive properties of the coupled assembly are also required. These may be obtained directly by experiment (as is done in this work), or predicted numerically. If a numerical model of the source (or the coupled assembly) is unavailable, a passive characterisation may be required. In this work, however, we will consider only the active characterisation of the source, i.e. the determination of acoustic free velocity.

The proposed characterisation requires a two-stage measurement procedure consisting of an operational phase and a passive phase, following which the source strengths (monopole volume velocities) are found by an inverse procedure. In the plane wave regime, as will be illustrated in the following sections, the source is represented by a single pair of monopoles. Beyond the planar regime this representation is extended by considering a pair of equivalent multipoles. The monopole and multipole regions are further extended in frequency by employing an energy approach for the highest part of the frequency range. The approach provides independent source strength data which retains both the phase and time structure of the acoustic signal (except in the energy regime where it will be seen that it can be reconstructed by employing simple assumptions) and is therefore suitable for auralisation purposes. A key advantage of the proposed equivalent source method is that all measurements are conducted in-situ, thus simplifying the experimental procedure.

The remainder of this paper is organised as follows. Section 2 will begin by introducing the equivalent source description used in this work, before formulating the proposed inverse characterisation procedure. Following this, section 3 will present an experimental case study where a high speed compressor unit is independently characterised through the proposed approach. Lastly, section 4 will draw some concluding remarks.

\section{Theory}

This section will begin by introducing the equivalent source description adopted in this work. Its justification, based on an equivalent field theorem, will be presented before the characterisation procedure itself is described.

\subsection{Equivalent Source Description and Justification}

The proposed characterisation procedure considers the in-duct acoustic source as a discrete array of volume velocity sources, i.e. monopoles. This equivalent source description is justified on the basis of an equivalent field theorem [14], as discussed below.

It is well known that equivalent electrical sources may be defined in terms of an open circuit voltage or a closed circuit current. Analogously, it has been shown that for a continuous composite elastic (source-receiver) system, the response field in a receiver sub-system, due to an arbitrary disturbance in the source sub-system, may be reproduced 
identically through an equivalent excitation at the coupling interface [14]. This equivalent excitation may be expressed in terms of either a blocked force or free velocity, both of which independently characterise the activity of the source sub-system. The blocked force has gained popularity within the structural dynamics community for the independent characterisation of vibration sources through the in-situ blocked force approach [13]. Similarly, Bobrovnitski and Pavic have made use of the blocked pressure to independently characterise acoustic sources [15].

In this paper, we will instead make use of the free velocity as an equivalent excitation. The free velocity is defined as the velocity at the coupling interface when the source is in operation but unloaded (i.e. a free boundary condition). It can be shown that if, in place of the initial excitation, this velocity is applied as a velocity difference between the source and receiver sub-systems, then the response field in the receiver is identical to that with the source in operation [14].

As an illustration, consider a monopole inside a duct in a plane wave regime: expansion and contraction of the monopole within the duct clearly creates a velocity difference in the fluid either side. In the equivalent source representation, a monopole is placed at the coupling interface between the source and receiver thereby creating a velocity difference between these domains. If the source strength is correctly chosen (so as to equal the free velocity of the source) then the response field in the receiver domain is identical to that occurring when the source is in operation, and the monopole can be said to be an equivalent source. This principle is recognised in [6]. In the following, this idea is extended beyond the plane wave regime by the use of multiple equivalent monopoles (i.e. a multipole). This approach requires the assumption that the continuous interface separating source and receiver can be adequately represented by a finite number of discreet sources, in other words, that it can be discretised. For completeness a derivation of the above notion is presented in Appendix A.

A key point regarding the equivalent field theorem is that the equivalent excitations are applied to the coupled system. This suggests that the equivalent excitations are obtainable from measurements made on the coupled assembly, and therefore in-situ. Doing so requires a two stage measurement procedure involving an active phase, with the source in operation, and a passive phase, with it switched off, as will be described in the following section.

\subsection{Inverse Characterisation Procedure}

Based on the above discussion and the developments of Appendix A, it is argued that an in-duct source may be independently described by its discretised acoustic free velocity, $\mathbf{v}_{\mathbf{f}}^{\mathbf{s}}$, and that this may, in theory, be obtained from measurements made in-situ on a coupled assembly. In this section we will consider the procedure necessary to experimentally determine the acoustic free velocity.

According to the results of Appendix A, the acoustic free velocity is the velocity difference, $\mathbf{v}_{\mathbf{a}_{2}}^{\mathbf{r}}-\mathbf{v}_{\mathbf{a}_{2}}^{\mathbf{s}}$ (where $s$ and $r$ refer to the source and receiver sub-systems, respectively, whilst $a$ denotes their coupling interface), that when applied to the interface of a coupled assembly (in the absence of an operational source) reproduces an identical response field in the receiver. Noting that a monopole is defined as a harmonically expanding rigid sphere which, by definition, imparts a velocity difference at its position, it is argued that the (discretised) free velocity may suitably be represented by an array of monopoles, and that the independent characterisation amounts to the determination of the strength, or volume velocity, of said monopoles. This may readily be achieved through an inverse procedure.

Suppose that the free velocity, $\mathbf{v}_{\mathbf{f}}^{\mathbf{s}}$, is approximated by $m$ monopoles, whose volume velocities are given by $u_{m}$. The unknown volume velocities contribute to a pressure field sampled at a number of positions, $p_{n}$. The contribution of each volume velocity to the pressure at a given point is determined by the frequency response function $(\mathrm{FRF}), H_{n m}$. From this we are able to construct the set of simultaneous equations,

$$
\begin{gathered}
p_{1}=H_{11} u_{1}+H_{12} u_{2}+\ldots+H_{1 m} u_{m} \\
p_{2}=H_{21} u_{1}+H_{22} u_{2}+\ldots+H_{2 m} u_{m} \\
\vdots \\
p_{n}=H_{n 1} u_{1}+H_{n 2} u_{2}+\ldots+H_{n m} u_{m} .
\end{gathered}
$$

The above may be cast into matrix form and, for $n=m$, providing that the FRF matrix is of full rank, a unique solution found through the inverse matrix $\mathbf{H}^{-\mathbf{1}} \in \mathbb{C}^{m \times m}$. Alternatively, for $n>m$, the Moore-Penrose pseudo inverse, $\mathbf{H}^{+} \in \mathbb{C}^{m \times n}$, may be used to establish the least squares solution [16]. 


$$
\left[\begin{array}{c}
u_{1} \\
u_{2} \\
\vdots \\
u_{m}
\end{array}\right]=\left[\begin{array}{cccc}
H_{11} & H_{12} & \ldots & H_{1 m} \\
H_{21} & H_{22} & \ldots & H_{2 m} \\
\vdots & \vdots & \ddots & \vdots \\
H_{n 1} & H_{n 2} & \ldots & H_{n m}
\end{array}\right]^{+}\left[\begin{array}{c}
p_{1} \\
p_{2} \\
\vdots \\
p_{n}
\end{array}\right]
$$

The volume velocity vector, $\mathbf{u} \in \mathbb{C}^{m}$, according to Appendix $\mathrm{A}$, is an independent property of the source sub-system, and may equally be thought of as a vector of acoustic free velocities. For clarity, however, these will be referred to hereafter as simply equivalent volume velocities.

Owing to its independence, the volume velocity vector may, in theory, be transferred between assemblies and used to predict their operational response, for example in the construction of a VAP. Note that this would require measurement or modelling of the coupled assembly's passive properties, which would implicitly include the passive properties of the source sub-system. Furthermore, it is worth noting that the spatially discretised nature of the interface velocity field not only negates the need to perform any modal decompositions, thus reducing the complexity of the measurement procedure and post processing, but allows for the characterisation to be performed in ducts of irregular geometry, as the duct geometry is implicitly accounted for by the FRF matrix, $\mathbf{H}$.

The experimental implementation of Eq. (2) requires a two part measurement procedure whereby the operational pressure vector, $\mathbf{p}$, and passive FRF matrix, $\mathbf{H}$, are determined. With the form of $\mathbf{H}$ determining the form of $\mathbf{u}$, the measured FRFs must relate pressure to volume velocity. Fortunately, FRF measurements of this type have been of interest to those within the fields of building acoustics [17] and NVH (Noise, Vibration and Harshness) [18, 19] for many years. This has led to the development of commercially available calibrated volume velocity sources [20,21] that are suitable for the measurement of the required FRFs.

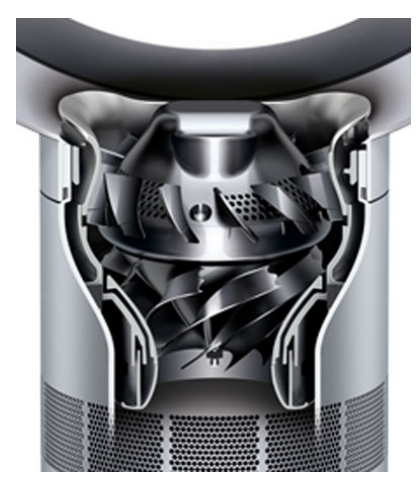

Figure 1: Illustration of the Dyson AM06 compressor unit used in the experimental validation of the proposed equivalent source method.

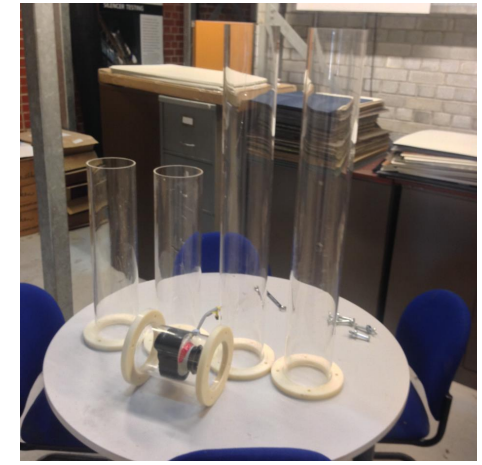

(a)

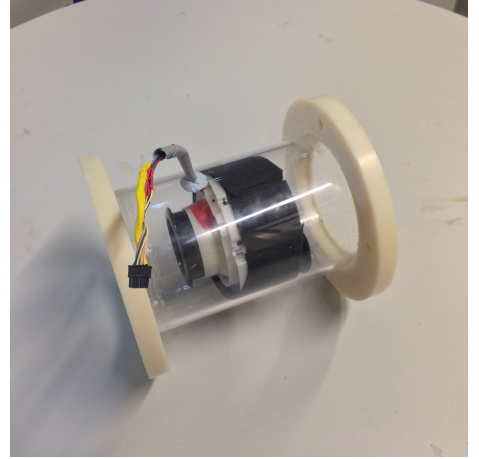

(b)

Figure 2: Assembly components, including compressor unit, sample holder and additional lengths of duct.

Whilst the above procedure may be applied directly in both planar and modal regimes (through monopole and multipole source descriptions, respectively) it is not sufficient to cover the entire audio range. To achieve a broadband characterisation it is necessary to employ an energy approach for the top end of the frequency range. The implementation of these three approaches will be discussed in the following section as part of an experimental case study.

\section{Experimental Study}

In what follows the above methods (equivalent monopole, multi-pole and energy) are introduced and applied to the characterisation of a compact compressor unit, an illustration of which is shown in figure 1.

It should be noted that the compressor unit was operated such that the effect of flow on the passive properties of the assembly was considered negligible [22]. As such, the required FRFs were able to be measured whilst the compressor was inactive. 


\subsection{Equivalent Monopoles}

We will begin by considering the characterisation in the planar region via the equivalent monopole approach. For the study considered here, where both inlet and outlet contributions are of interest, the equivalent kinematic excitation is approximated by two monopoles, one spaced either side of the source in question, as shown in figure 3 . Consequently, in the planar region, the source is characterised by two volume velocities (or equivalently, acoustic free velocities). Eq. (2) thus takes the form,

$$
\left[\begin{array}{l}
q_{1} \\
q_{2}
\end{array}\right]=\left[\begin{array}{cc}
H_{11} & H_{12} \\
H_{21} & H_{22} \\
\vdots & \vdots \\
H_{n 1} & H_{n 2}
\end{array}\right]^{+}\left[\begin{array}{c}
p_{1} \\
p_{2} \\
\vdots \\
p_{n}
\end{array}\right] .
$$

With two unknowns, Eq. (3) requires two operational pressures, along with their respective FRFs, in order to establish a determined solution. Additional pressures and FRFs may be included to over-determine the problem.

\subsubsection{Experimental Set-up and Procedure}

The experimental set-up used here, and throughout the remainder of this paper, is shown diagrammatically in figure 3. The compressor unit was housed within a short section of duct, the entirety of which is considered the source sub-system. Additional lengths of duct are attached to either side of the source sub-system so as vary the properties of the receiver sub-system. Three assemblies, $\mathbf{A}, \mathbf{B}$ and $\mathbf{C}$, were constructed, details of which are given in table 1 . The varying acoustic load of each assembly allows us to illustrate the independence of the equivalent volume velocities. Shown in figure 2 are some of the test rig components used to construct these assemblies.

\begin{tabular}{cccc} 
Assembly & Inlet $(\mathrm{cm})$ & Sample Holder $(\mathrm{cm})$ & Outlet $(\mathrm{cm})$ \\
\hline A & 61 & 15 & 61 \\
B & 33 & 15 & 29 \\
C & 33 & 15 & 61
\end{tabular}

Table 1: Assembly details. All ducts have inner diameter of $14 \mathrm{~cm}$.

It was assumed that the chosen lengths of duct provided enough variation in the acoustic loading without introducing additional aerodynamic loading, and that the sound generating mechanisms of the source did not vary between assemblies. Note however, that if the aerodynamic loading of the target installation (i.e. the assembly whose operational response we would like to predict) is time invariant, and the source is characterised under an appropriate (aerodynamic) load, the proposed method theoretically remains applicable.

The position of the equivalent monopoles were taken to be at the centre of the duct, approximately $2.5 \mathrm{~cm}$ from the compressor's furthest extremities. For each assembly four low noise measurement microphones were placed about the inlet and outlet, as in figure 3. The two part characterisation procedure was as follows;

- The compressor was turned off and the FRF matrix, $\mathbf{H}$, was measured between all eight microphone positions and the two equivalent monopole positions.

- The compressor was then turned on and the external pressure, $\mathbf{p}$, measured at all 8 microphones.

The operational pressures were recorded in the time domain and post processing carried out in MATLAB using a hybrid time/frequency domain approach (see Appendix B). This approach was adopted so as to retain the time structure of the operational compressor, allowing for realistic auralisations to be reproduced at a later date. 


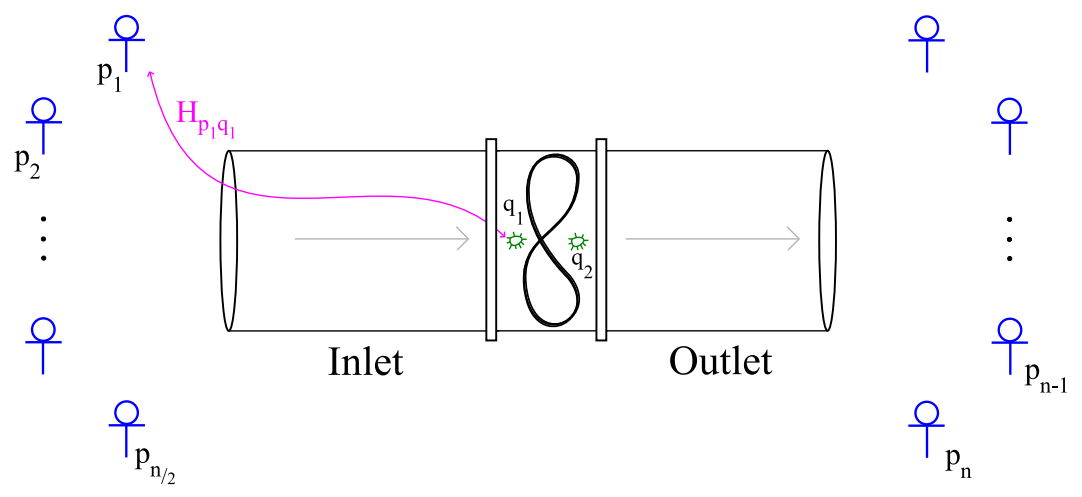

Figure 3: General experimental set-up for equivalent source characterisation.

The region over which the equivalent monopole approach is expected to yield an appropriate source characterisation is dictated by the cross sectional diameter of the assembly, i.e. the cut-on of higher order modes. Shown in table 2 are the estimated cut-on frequencies for the first 12 modes of a circular duct with an internal diameter of $14 \mathrm{~cm} \mathrm{[23].}$ From this we are able to estimate the upper limit of the equivalent monopole approach as approximately $1.4 \mathrm{kHz}$.

\begin{tabular}{c|cccc}
$m \backslash n$ & 0 & 1 & 2 & 3 \\
\hline 0 & 0 & 2988 & 5471 & 7934 \\
1 & 1436 & 4158 & 6657 & 9129 \\
2 & 2382 & 5230 & 7775 & 10271 \\
3 & 3276 & 6251 & 8848 & 11375 \\
4 & 4157 & 7239 & 9890 & 12450
\end{tabular}

Table 2: Cut-on frequencies $(\mathrm{Hz})$ for a circular duct of internal diameter $d=1.4 \mathrm{~cm}(m=$ azimuthal order $; n=$ radial order $)$.

\subsubsection{Results and Validation}

Shown in figure 4 are a set of inlet and outlet volume velocities obtained from assembly A. Note that all results presented hereafter have been normalised to an unspecified value for the purpose of confidentiality. Each colour plot represents a determined solution calculated using a different pair of operational pressures, each located at one end of the assembly. In black are the solutions of the over-determined problem. These were calculated using all 8 operational pressures. It can be seen that the volume velocities determined from each microphone pair are in excellent agreement with the over-determined solution up to approximately $1.4 \mathrm{kHz}$. Above this, agreement begins to deteriorate with questionable artefacts appearing in the determined volume velocities. This suggests that above 1.4 $\mathrm{kHz}$ the continuous kinematic excitation is not sufficiently approximated by a single pair of monopoles. This is in agreement with the predicted cut-on of higher order modes.

Shown in figure 5 are the over-determined inlet and outlet volume velocities obtained from each assembly. Whilst the operational pressure varies significantly from one assembly to the next, due to the different lengths of duct, the variation across the set of volume velocities obtained from each assembly (figure 5) is significantly lower. This provides experimental support to the theory that the acoustic free velocity is an intrinsic property of the source and independent of the receiver properties. Furthermore, it suggests that the acoustic loading by the receiver can be removed effectively by the inverse procedure employed. Interestingly, the independence of the source appears to hold even above the cut-on of the first azimuthal mode at around $1.4 \mathrm{kHz}$. 


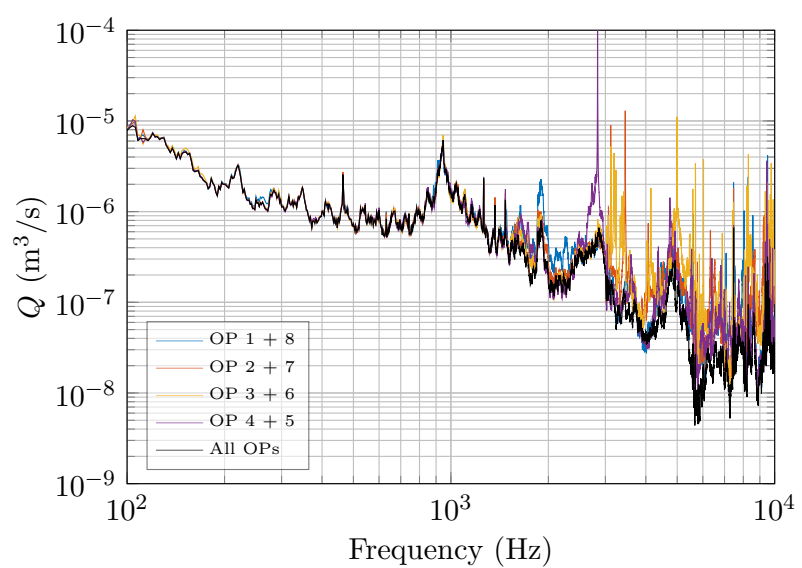

(a)

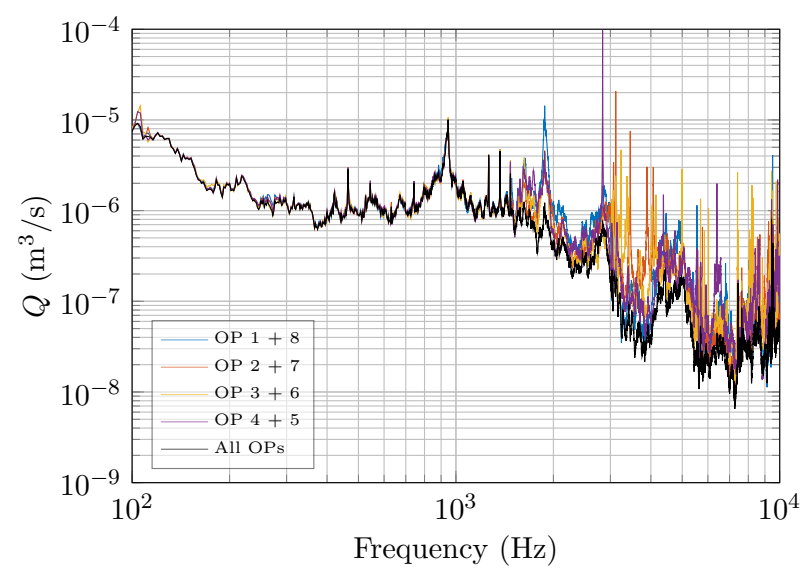

(b)

Figure 4: Equivalent volume velocities obtained from assembly A - a) Inlet velocities, b) Outlet velocities. Coloured plots correspond to the determined solutions, found using a single pair of operational pressure measurements on the source and receiver side (OP $\mathrm{i}=$ operational pressure at position i). Black plots correspond to the over-determined solutions, where all 8 operational pressure measurements were used.

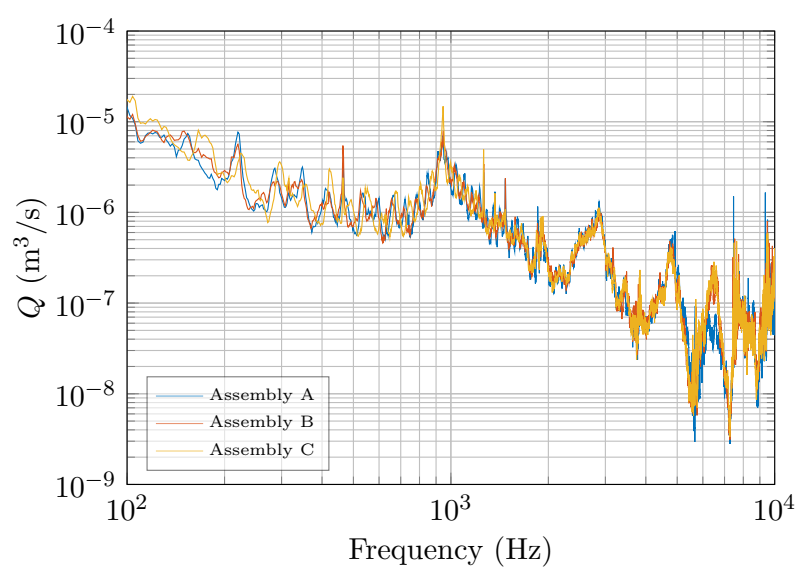

(a)

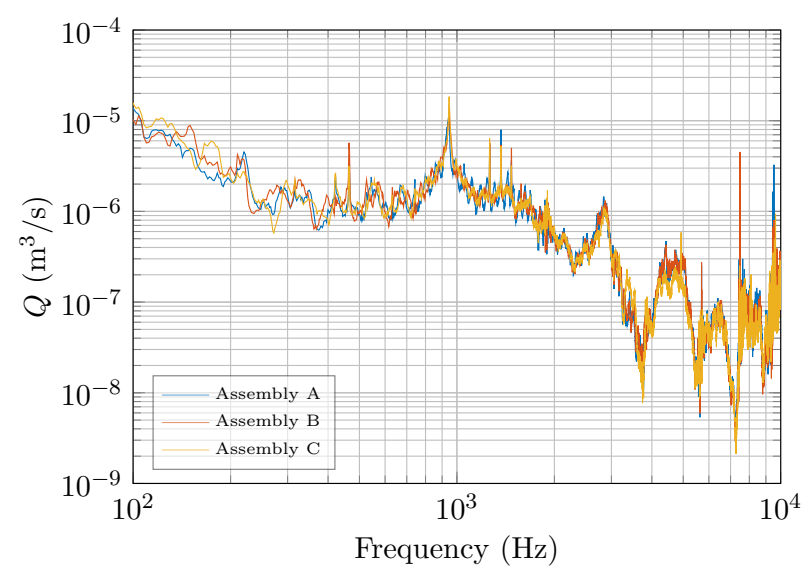

(b)

Figure 5: Over-determined inlet and outlet source strengths obtained from assembly A, B and C - a) Inlet velocities, b) Outlet velocities.

Whilst figure 5 demonstrates the independence of the acquired volume velocities, it offers no indication of their quality, nor their ability to predict the operational pressure of an assembly. As a means of assessing the above, two validation procedures are performed; an on-board validation and a transferability validation.

An on-board validation involves the prediction of an operational pressure using volume velocities obtained from the same assembly in which the prediction is made. The on-board validation is performed as per Eq. (4), where; $p_{r_{i n}}$ and $p_{r_{\text {out }}}$ are a pair of reference pressures located towards the inlet and outlet, respectively, and $q_{1}$ and $q_{2}$ are the over-determined volume velocities,

$$
\left[\begin{array}{c}
p_{r_{\text {in }}} \\
p_{r_{\text {out }}}
\end{array}\right]_{A}=\left[\begin{array}{ll}
H_{r_{\text {in }} q_{1}} & H_{r_{\text {in }} q_{2}} \\
H_{r_{\text {out }} q_{1}} & H_{r_{\text {out }} q_{2}}
\end{array}\right]_{A}\left[\begin{array}{l}
q_{1} \\
q_{2}
\end{array}\right]_{A}
$$

where subscript $A$ refers to assembly $A$. It is important to note that the reference pressures are not used in the determination of the volume velocities, but are measured simultaneously to those that are. 
The transferability validation checks whether the free velocities measured on one assembly, say assembly A, can be used to predict the operational pressures measured on another, say assembly B, as in Eq. (5).

$$
\left[\begin{array}{c}
p_{r_{\text {in }}} \\
p_{r_{\text {out }}}
\end{array}\right]_{B}=\left[\begin{array}{ll}
H_{r_{\text {in }} q_{1}} & H_{r_{\text {in }} q_{2}} \\
H_{r_{\text {out }} q_{1}} & H_{r_{\text {out }} q_{2}}
\end{array}\right]_{B}\left[\begin{array}{l}
q_{1} \\
q_{2}
\end{array}\right]_{A}
$$

Shown in figure 6 is the on-board validation of assembly A. Measured and predicted pressures are shown in orange and blue, respectively. Whilst a total of eight operational pressures were measured, predictions were made using overdetermined volume velocities calculated from six operational pressures. The remaining two pressures were used as references for the on-board validation. It can be seen that good agreement is obtained across the entire planar region. Beyond approximately $1.4 \mathrm{kHz}$ the prediction begins to fall short of the measured response. This suggests that some source of energy has not been accounted for, i.e. high order modal contributions.

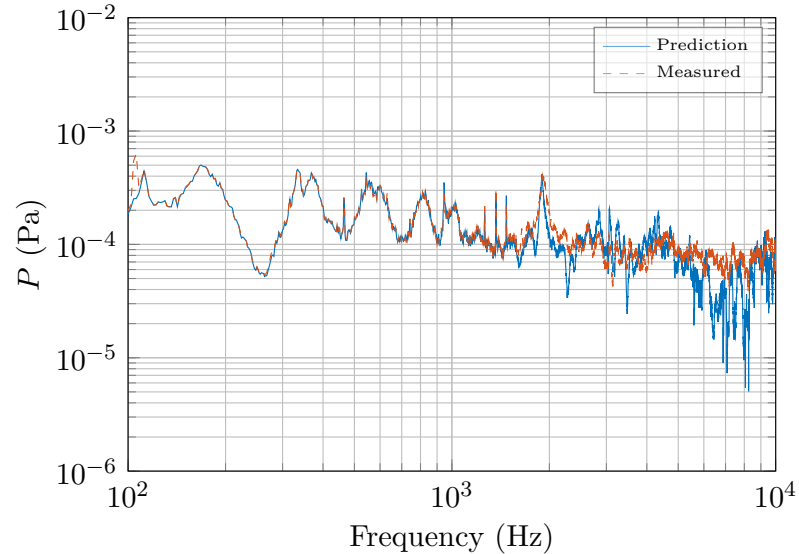

(a)

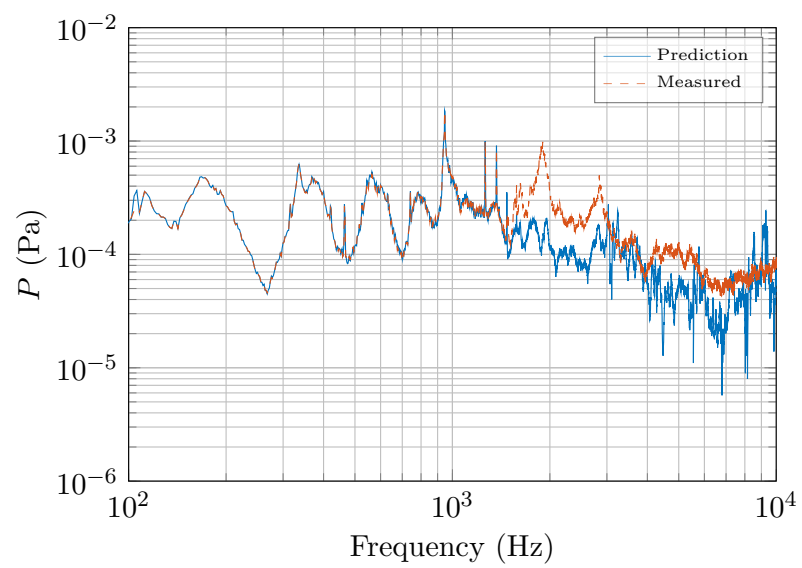

(b)

Figure 6: On-board validation - using equivalent monopole volume velocities obtained from assembly A to predicted the reference operational pressures of assembly A - a) Inlet pressure, b) Outlet pressure.

Shown in figure 7 is the transferability validation of assembly A. Here, the volume velocities obtained from assembly $\mathbf{A}$ are transferred to assembly $\mathbf{B}$, and used to predict its operational response. Again, good is agreement obtained up to approximately $1.4 \mathrm{kHz}$, beyond which the predictions fall short.

The above results clearly demonstrate that the volume velocities, obtained through the proposed approach, are able to predict the operational pressure response in both initial and secondary assemblies, in the planar region, with a high degree of accuracy. 


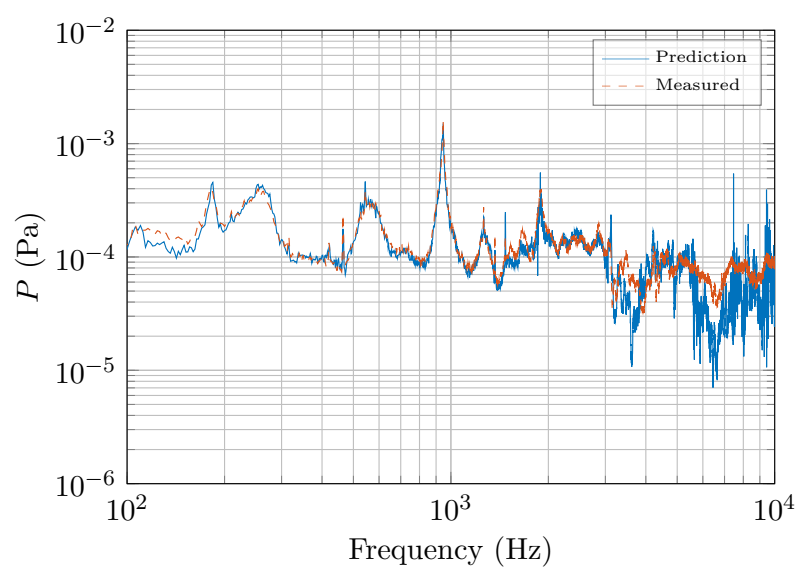

(a)

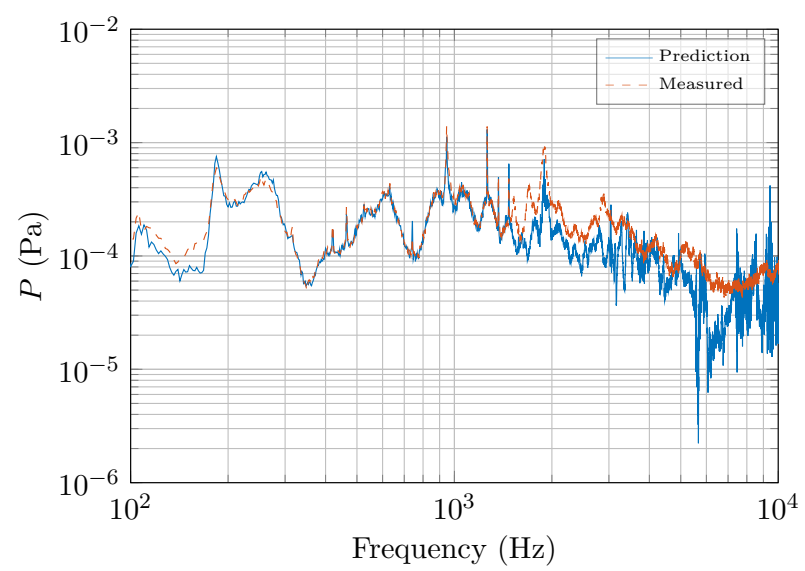

(b)

Figure 7: Transferability validation - using equivalent monopole volume velocities obtained from assembly A to predicted the reference operational pressures of assembly $\mathbf{B}$ - a) Inlet pressure, b) Outlet pressure.

\subsection{Equivalent Multipole}

We will now consider the characterisation in the modal region via the equivalent multipole approach. The equivalent multipole is the logical progression from the equivalent monopole, where the source is now approximated by an array of equivalent monopoles, in other words by a multipole. For the example considered here, where both inlet and outlet contributions are of interest, the kinematic excitation is approximated by two multipoles, one spaced either side of the source.

Unlike the planar region, the modal region is made up of multiple propagating modes, the orders of which extend to infinity. As such, one must limit interest to a particular range of modes such that the characterisation is experimentally viable. Eq. (2) thus takes the form,

$$
\left[\begin{array}{c}
\mathbf{q}_{\text {in }} \\
\mathbf{q}_{\text {out }}
\end{array}\right]=\left[\begin{array}{cc}
\mathbf{H}_{\text {in,in }} & \mathbf{H}_{\text {in,out }} \\
\mathbf{H}_{\text {out,in }} & \mathbf{H}_{\text {out }, \text { out }}
\end{array}\right]^{+}\left[\begin{array}{c}
\mathbf{p}_{\text {in }} \\
\mathbf{p}_{\text {out }}
\end{array}\right]
$$

where; $\mathbf{q}_{\text {in }} \in \mathbb{C}^{n}$ and $\mathbf{q}_{\text {out }} \in \mathbb{C}^{n}$ are the inlet and outlet volume velocities, respectively, $\mathbf{p}_{\text {in }} \in \mathbb{C}^{m}$ and $\mathbf{p}_{\text {out }} \in \mathbb{C}^{m}$ are the inlet and outlet external pressures, respectively, and $\mathbf{H}_{\mathbf{x}, \mathbf{y}} \in \mathbb{C}^{n \times m}$ are the corresponding FRF matrices. It is assumed that the order of the multipole, $n$, should correspond to the number of modes in the frequency range of interest. If satisfied, the condition $m \geq n$ is then required for a determined solution.

For the example considered here, each multipole is composed of 5 monopoles, $n=5$. As only 10 operational pressures were measured $(m=n)$, the results presented hereafter are those of the determined solution only.

An important consideration when implementing the multipole approach is the positioning of the individual monopoles, as alignment with nodal lines would likely suppress the contribution of any corresponding modes. Previous authors have considered the optimisation of microphone positioning for duct decomposition schemes [24] and similar precautions may need to be taken into consideration when implementing the multipole approach. In this work, however, it was assumed that the modal field within the duct was in rotation and therefore the monopole positioning was not considered an issue.

\subsubsection{Experimental Set-up and Procedure}

The experimental set-up and characterisation procedure follows that of the equivalent monopole approach discussed in section 3.1.1, whilst accounting for the additional monopole positions and the 10 external microphones. The multipole configuration used is shown figure 8 .

Given the configuration of monopoles used, based on 2 points per spatial wave length it should be possible to resolve the modes $(0,0),(1,0),(0,1),(2,0),(1,1)$ and $(2,1)$. The first radial mode is resolved since we have included a central monopole position and 4 outer radial positions. The first two azimuthal modes are resolved since the 4 outer 
radial monopole are spread around the circumference of the duct. The first propagating mode that can not be resolved given the multipole configuration is thought to be the azimuthal mode $(3,0)$ at a frequency of $3.2 \mathrm{kHz}$ (see table 2). To resolve this mode an additional radial monopole would be required.

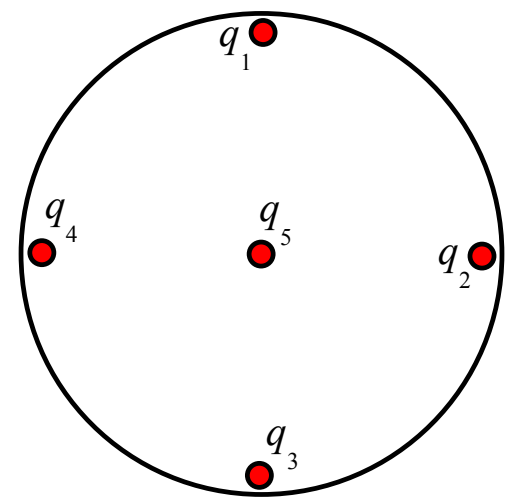

Figure 8: Duct crosss section showing the monopole positioning used in the multipole approach.

\subsubsection{Results and Validation}

With 10 equivalent source positions having been utilised it is difficult to compare the acquired volume velocities against those obtained from a second assembly. Instead, validation of the multipole approach will be achieved through a transferability validation, as in section 3.1.2.

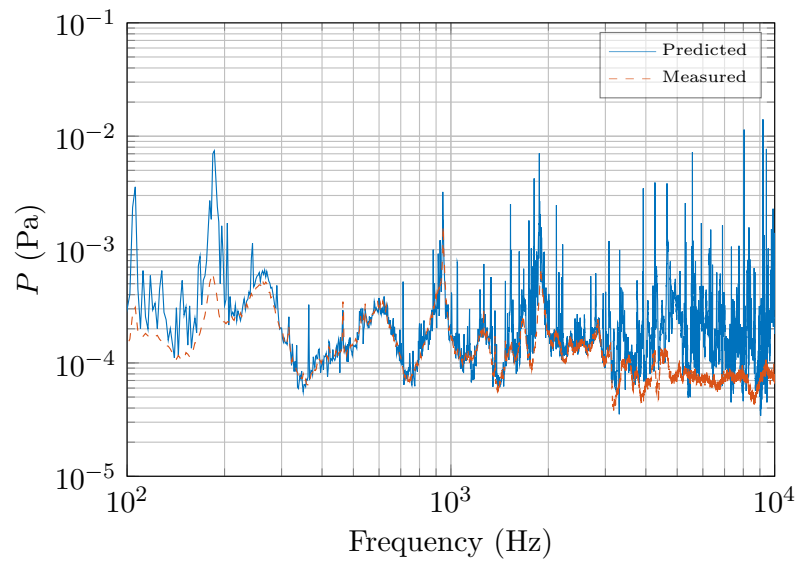

(a)

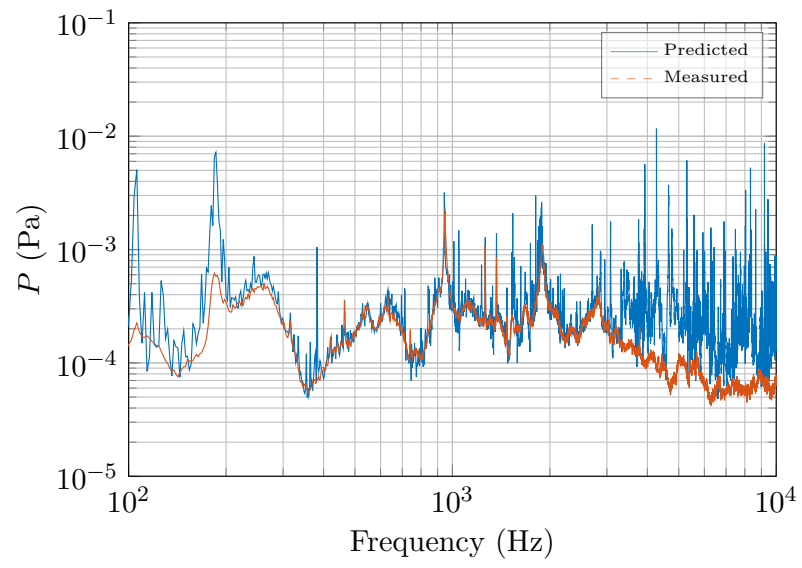

(b)

Figure 9: Transferability validation - using equivalent multipole volume velocities obtained from assembly $\mathbf{A}$ to predict the reference operational pressures of assembly $\mathbf{B}$ - a) Inlet pressure, b) Outlet pressure. 


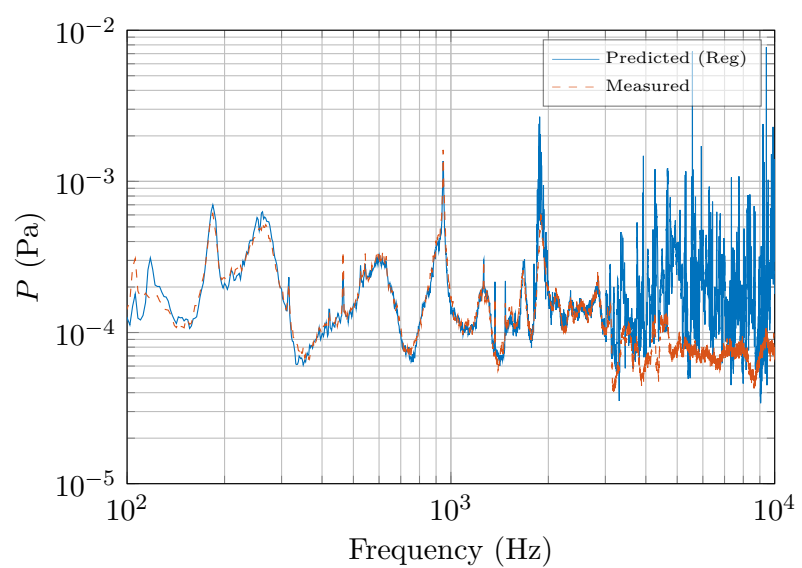

(a)

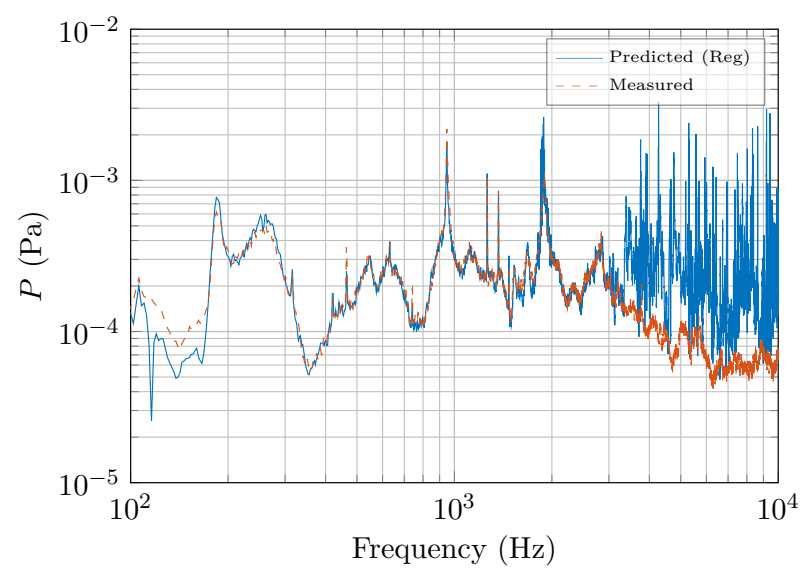

(b)

Figure 10: Transferability validation - using regularised equivalent multipole volume velocities obtained from assembly $\mathbf{A}$ to predict the reference operational pressures of assembly B - a) Inlet pressure, b) Outlet pressure.

Shown in figure 9 are the measured and predicted external pressures of assembly B. Predictions were made using a set of determined volume velocities obtained from assembly A, as per Eq. (5). Results are clearly affected by what is likely inversion error due to the ill-conditioning of the larger $10 \times 10$ FRF matrix. Methods such as over-determination and regularisation are often used in an attempt to reduce error of this nature $[25,26]$.

To illustrate that this error is not a limitation of the method, some regularisation is applied to the inverse procedure. The results shown in figure 10 correspond to those of figures 9 with the addition of some basic regularisation, where any singular values occurring below an absolute threshold of $10^{-6}$ were discarded. This value was chosen arbitrarily such that the predictions were in reasonable agreement with the measurements.

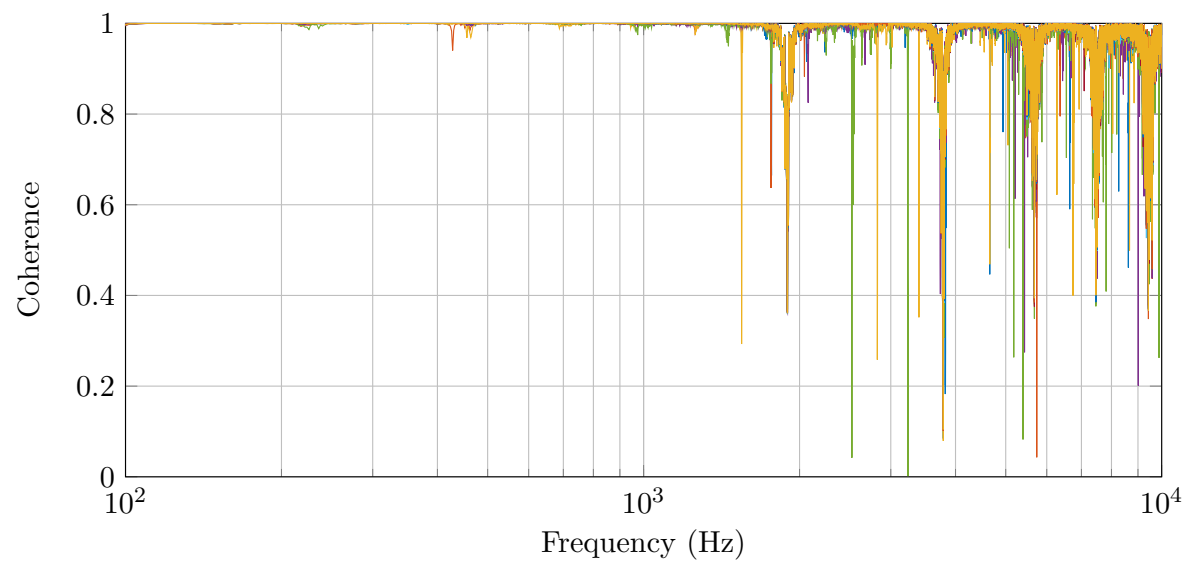

Figure 11: Coherences obtained between volume velocity source and 10 external microphones during the measurement of the multipole FRF matrix.

The regularized predictions agree well with measurements up to approximately $3.2 \mathrm{kHz}$, i.e. the cut-on of the $3 \mathrm{rd}$ azimuthal mode. Above this, regularisation has little to no effect. This suggests that the additional modes occurring above $3.2 \mathrm{kHz}$ are partly to blame, which aligns well with the predicted modal frequencies given the number of equivalent sources. Whilst over-determination can be expected to reduce the severity of inversion errors, it cannot compensate for an insufficient order of multipole in the equivalent source and so results above $3.2 \mathrm{kHz}$ are expected to remain erroneous. 
A noticeable error is observed in both the inlet and outlet predictions around approximately $2 \mathrm{kHz}$. This error is a result of poor coherence between the volume velocity source and the external microphones during the FRF measurements. An example of this coherence is shown in figure 11. The poor coherence is a result of the limited dynamic range of the internal volume velocity microphone. This could be improved with the use of a microphone with a lower noise floor.

The above results confirm that the multipole representation is able to extend the frequency range compared with that of the monopole representation. However, the maximum frequency remains limited by the order of the multipole which can only represent the behaviour of a comparable number of duct modes, in this case, five monopoles each side was sufficient up to $3.2 \mathrm{kHz}$. Characterisation beyond this, say up to $10 \mathrm{kHz}$, would require a significantly higher order multipole, approximately equal to the mode count up to this frequency, and this is not practicable within reasonable equipment requirements. Therefore, to complete the source characterisation up to $10 \mathrm{kHz}$ an alternative approach is required as discussed in the next section.

\subsection{Equivalent Energy}

It would not be unreasonable to expect the synthesis of realistic auralizations, for example in the construction of a VAP, to require predictions up to and perhaps beyond $10 \mathrm{kHz}$. If such is the case, the multipole characterisation approach may no longer be suitable, be it due to limited hardware, an insufficient source definition, or numerical instability.

It is proposed that such an issue may, however, be circumvented by considering the problem from an energy standpoint. This may be justified on the basis that at high frequencies the modal behaviour of the assembly is complex enough that the phase between any two modes may be considered random, similar to the concept of a diffuse field. In this region phase may be discarded and the inverse procedure carried out using magnitudes only. It is further proposed the FRF matrix relating the multipole source and microphone array be averaged,

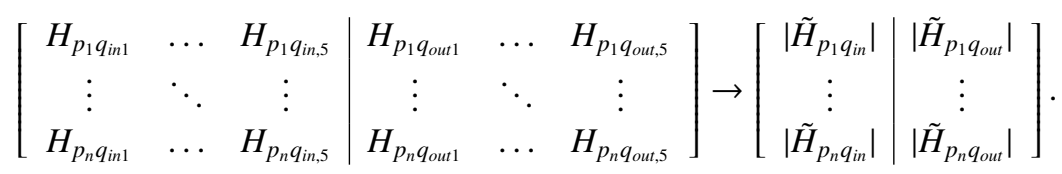

such that a pair of equivalent monopoles are defined. The spatial averaging of the FRF matrix is conceptually similar to the spatial assumptions employed in Statistical Energy Analysis (SEA) [27], and the mobility averaging employed in the reception plate method, a power based approach to characterisation of structural sources [28].

The high frequency contribution of the source is thus approximated by two equivalent 'energy' sources,

$$
\left[\begin{array}{c}
\hat{q}_{\text {in }} \\
\hat{q}_{\text {out }}
\end{array}\right]=\left[\begin{array}{c|c}
\left|\tilde{H}_{p_{1} q_{\text {in }}}\right| & \left|\tilde{H}_{p_{1} q_{\text {out }}}\right| \\
\vdots & \vdots \\
\left|\tilde{H}_{p_{n} q_{\text {in }}}\right| & \left|\tilde{H}_{p_{n} q_{\text {out }}}\right|
\end{array}\right]^{+}\left[\begin{array}{c}
\left|p_{1}\right| \\
\vdots \\
\left|p_{n}\right|
\end{array}\right]
$$

where $\left|\tilde{H}_{p_{1} q_{i n}}\right|$, for example, represents the magnitude of the complex averaged FRF between the 5 inlet multipole positions and the external pressure at microphone 1 , and $\hat{q}_{\text {in }}$ and $\hat{q}_{\text {out }}$ are real valued source strengths based on the equivalent energy.

It should be noted that any subsequent auralization would require the reintroduction of a random phase to the spectrum of the equivalent energy source. This may be achieved by multiplying the source term by a complex exponential of the form,

$$
\left[\begin{array}{l}
q_{1} \\
q_{2}
\end{array}\right]=\left[\begin{array}{l}
\hat{q}_{1} \\
\hat{q}_{2}
\end{array}\right] e^{i\left[\theta_{1} \theta_{2}\right]^{T}}
$$

where $\theta$ is a randomly generated phase angle [29].

\subsubsection{Experimental Set-up and Procedure}

The equivalent energy approach does not require any additional measurements to be made further to the multipole characterisation, and may be applied in the post processing of the multipole measurement data.

In this study, the $10 \times 10 \mathrm{FRF}$ matrix used in section 3.2 is averaged, according to Eq. (7), to a $10 \times 2$ magnitude only FRF matrix. The equivalent energy sources are subsequently determined as per Eq. (8). 


\subsubsection{Results and Validation}

Validation of the energy based approach is achieved through a transferability validation. Shown in figure 12 are the measured and predicted external pressures of assembly B. Predictions were made using the equivalent energies determined from assembly A. It can be seen that although there are significant discrepancies in the lower frequency range, as expected due to the neglect of phase, above approximately $1-2 \mathrm{kHz}$ agreement is quite good, certainly better than that of the equivalent monopole and multipole approaches.

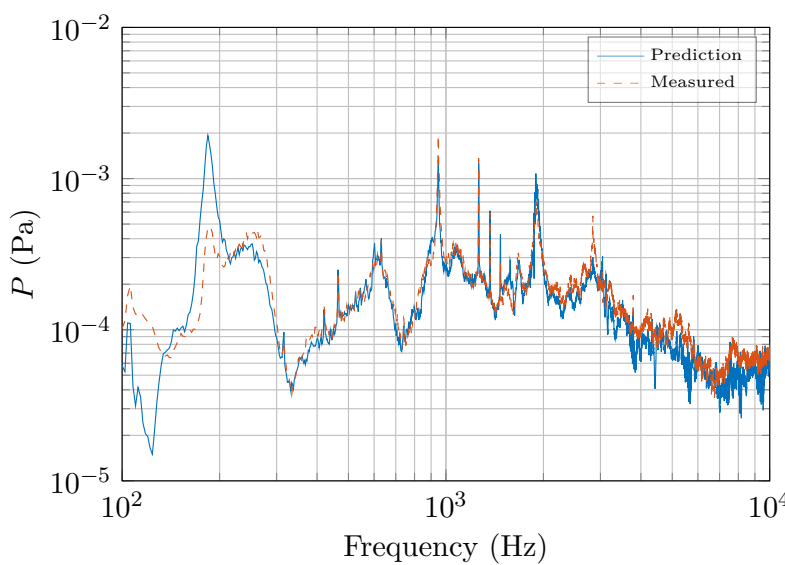

(a)

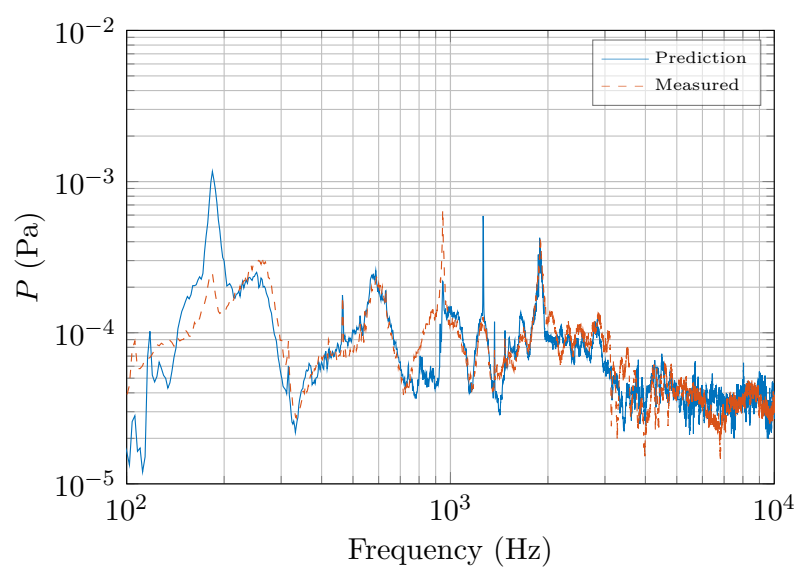

(b)

Figure 12: Transferability validation - using equivalent energy volume velocities obtained from assembly $\mathbf{A}$ to predicted the reference operational pressures of assembly $\mathbf{B}$ - a) Inlet pressure, b) Outlet pressure.

The results suggest that through simple post processing, a high frequency characterisation can be achieved that yields an independent and transferable source quantity, at no additional experimental effort over and above the multipole approach presented in section 3.2 .

\subsection{Broadband Characterisation}

Using a combination of the above procedures (equivalent monopole up to the first cut-on, equivalent multipole up to the $n$th cut-on, and the energy approach beyond) it is possible achieve a broadband and independent source characterisation using the equivalent field representation.

Shown in figure 13 are the measured and predicted external pressures of assembly $\mathbf{B}$. Predictions were obtained through a combination of the 3 methods proposed above, with equivalent volume velocities obtained from assembly A. No smoothing was used between the predictions of each regime. Excellent agreement is obtained from $100 \mathrm{~Hz}$ to $10 \mathrm{kHz}$ with important spectral characteristics captured. Some small errors can, however, be seen in the low frequency prediction, roughly between $100-200 \mathrm{~Hz}$. This is likely due to experimental error as these results were obtained from the first attempt at the multipole characterisation and it is believed these results could be improved. It should be noted that the low frequency (equivalent monopole) results used in the combined prediction are not the same as those presented in section 3.1. The low frequency results presented in figure 13 have been obtained from the equivalent multipole measurements presented in section 3.2 by using only the central source positions. This was due to a change in the positioning of the compressor within the sample holder between the initial monopole and multipole characterisations. Nevertheless, the results clearly illustrate the potential application of the proposed source characterisation methods. 


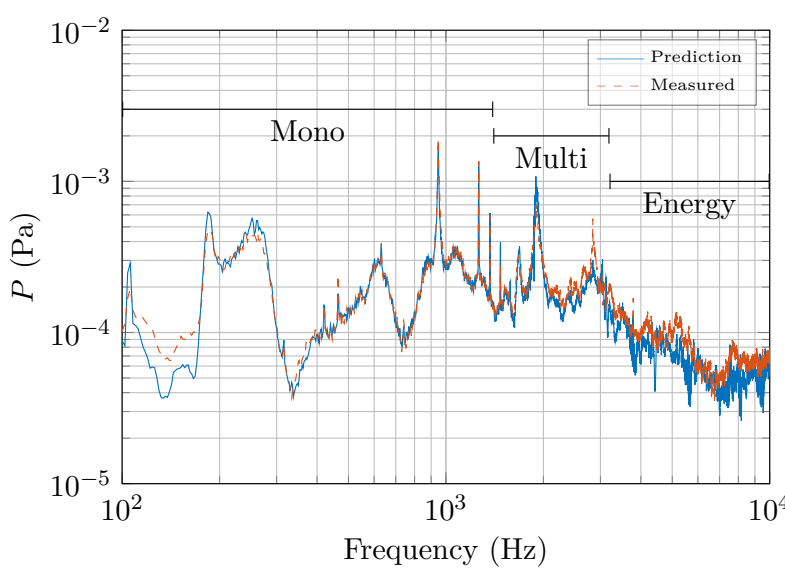

(a)

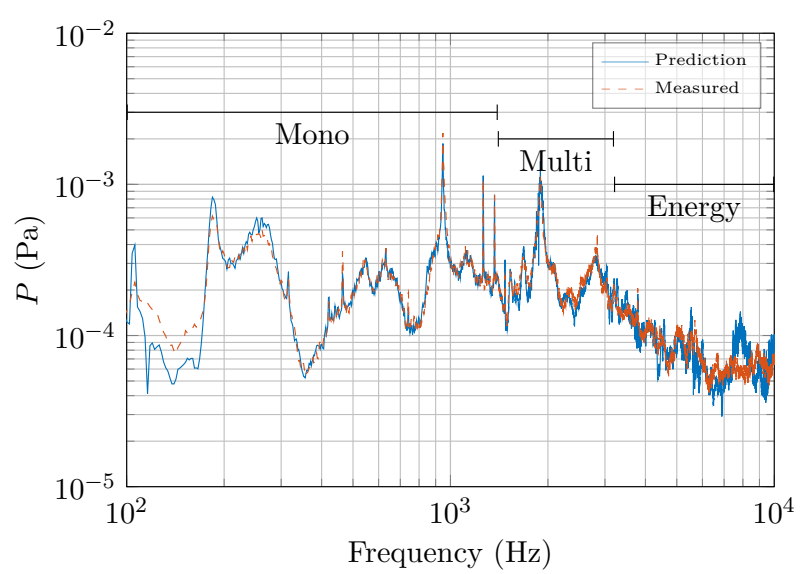

(b)

Figure 13: Transferability validation - using a combination of equivalent volume velocities obtained from assembly $\mathbf{A}$, via each of the proposed methods, to predicted the reference operational pressures of assembly $\mathbf{B}$ - a) Inlet pressure, b) Outlet pressure.

\section{Conclusions}

The purpose of this study was to develop an experimental method for independently characterising low Mach number fluid machines, such as pumps, fans, etc., for in-duct applications. This was achieved through the application of an equivalent field representation. Based on the discretisation of a coupling source-receiver interface it was shown that an acoustic source may be independently characterised by its free velocity, and that this may be represented by a discrete array of monopoles. Following a two part measurement procedure, an inverse method is used to determine the strengths of these monopoles, thus independently characterising the source.

Implementation of the proposed characterisation was illustrated through an experimental case study. In order to provide a broadband characterisation 3 complimentary methods were established.

It was shown that an equivalent monopole approach provides independent and transferable data suitable for operational predictions up to the cut-on of the first azimuthal mode. It was further shown that the frequency range may be extended through the use of an equivalent multipole. Due to the larger matrix inversion and lack of over-determination the results where contaminated by inversion error. This was suppressed using some basic regularization and an acceptable result obtained up to the cut-on frequency of the $n=2, m=3$ mode. In an attempt to avoid the need for additional instrumentation at high frequencies an alternative energy based approach was introduced. High frequency predictions appeared to follow the general trend of the measured response. A combination of the three methods presented offers the potential for a source characterisation that extends the entire frequency range of interest.

\section{Acknowledgements}

This project was co-funded by Innovate UK. The University of Salford and Dyson Technology Ltd would like to thank Innovate UK for their support throughout this project

\section{Appendix A. Equivalent Field Representation}

This section will introduce the equivalent field representation and discuss its application to the independent characterisation of in-duct acoustic sources.

It was shown by Bobrovnitskii [14] that the response field of a receiver sub-system in a composite elastic system, due to an external force acting on a coupled source sub-system, may be reproduced identically through an equivalent kinematic excitation at the source-receiver interface, whilst the external force is inactive. This equivalent field representation will allow for the formulation of an inverse procedure suitable for the independent characterisation of 
in-duct sources. For completeness a derivation of the appropriate theory is presented here. This may be considered a special case of Bobrovnitskii's theorem and the reader is referred to [14] for a full account of the theory in a more general form.

For those familiar with the theorem presented in [14], its application in the current context (i.e. acoustic source characterisation) may be justified as so; the original theory of [14] is formulated in terms of differential operators and force densities, as such it applies generally to continuum interfaces, such as those surrounding an acoustic source. The translation from force densities to pressure can be made on the basis that pressure is analogous to normal stress, and that fluids do not support shear stress. Since we consider only the pressure over a $2 \mathrm{~d}$ plane (i.e. the in-duct application), the notion of a stress tensor reduces to the normal stress component and consequently a scalar pressure. Further confidence in the application of this theorem to an acoustic case is gained from [15], where Bobrovnitskii's 2nd representation (i.e. a blocked pressure relation) is used to characterise an acoustic source.
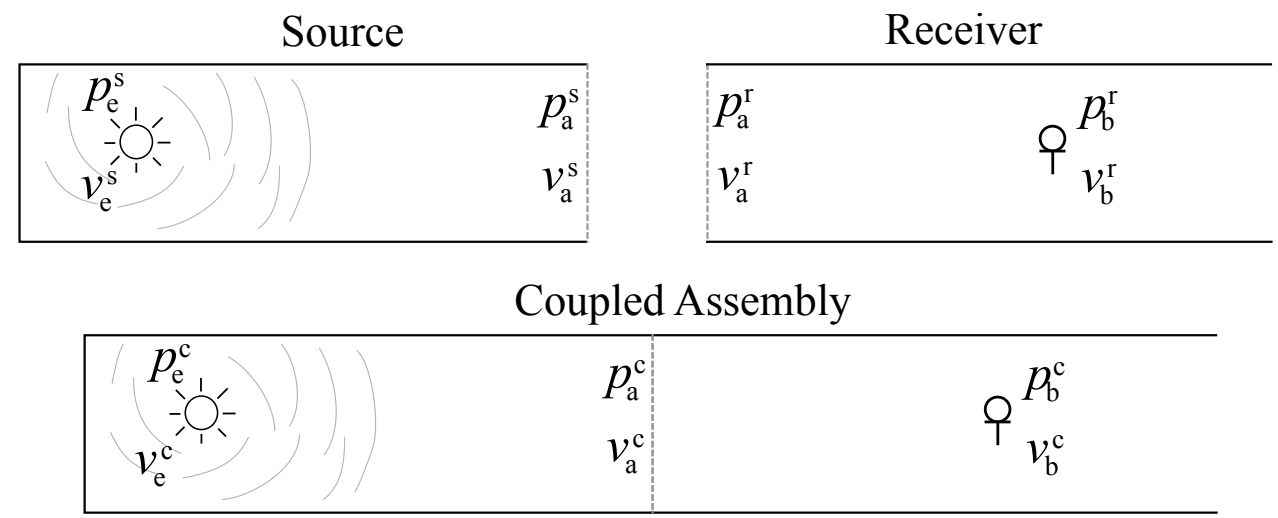

Figure A.14: Assembly schematic

\section{Appendix A.1. General Case}

Let us begin by considering the assembly depicted in figure A.14, where some set of unknown pressure sources, $\mathbf{p}_{\mathbf{e}}^{\mathbf{s}}(\omega) \in \mathbb{C}^{\mathbb{M}}$, act on the internal degrees of freedom (DoFs), $e$, within the source sub-system (denoted s) of a sourcereceiver assembly.

In what follows it is assumed that the continuous interface separating the source and receiver sub-systems, denoted here by $a$, may be approximated, to an arbitrary level of confidence, through its discretisation. That is, the interface may be described approximately by a finite number of DoFs. Consequently, the pressure and velocity fields over the interface are given, approximately, by the vectors $\mathbf{p}_{\mathbf{a}}(\omega) \in \mathbb{C}^{N}$ and $\mathbf{v}_{\mathbf{a}}(\omega) \in \mathbb{C}^{N}$, respectively, where $N$ is the number of DoFs used in the discretisation.

Assuming a linear and time invariant system and taking into account the discretisation of the coupling interface, Eqs. (A.1)-(A.4) describe generally the pressure-velocity relationships of the two uncoupled sub-systems, $\mathbf{s}$ and $\mathbf{r}$,

$$
\begin{aligned}
& \mathbf{p}_{\mathrm{e}}^{\mathrm{s}}=\mathbf{Z}_{\mathrm{ea}}^{\mathrm{s}} \mathbf{v}_{\mathrm{a}}^{\mathrm{s}}+\mathbf{Z}_{\mathrm{ee}}^{\mathrm{s}} \mathbf{v}_{\mathrm{e}}^{\mathrm{s}} \\
& \mathbf{p}_{\mathrm{a}}^{\mathrm{s}}=\mathbf{Z}_{\mathrm{aa}}^{\mathrm{s}} \mathbf{v}_{\mathrm{a}}^{\mathrm{s}}+\mathbf{Z}_{\mathrm{ae}}^{\mathrm{s}} \mathbf{v}_{\mathrm{e}}^{\mathrm{s}} \\
& \mathbf{p}_{\mathrm{a}}^{\mathbf{r}}=\mathbf{Z}_{\mathrm{aa}}^{\mathrm{r}} \mathbf{v}_{\mathrm{a}}^{\mathbf{r}}+\mathbf{Z}_{\mathrm{ab}}^{\mathrm{r}} \mathbf{v}_{\mathrm{b}}^{\mathrm{r}} \\
& \mathbf{p}_{\mathrm{b}}^{\mathbf{r}}=\mathbf{Z}_{\mathrm{ba}}^{\mathbf{r}} \mathbf{v}_{\mathrm{a}}^{\mathbf{r}}+\mathbf{Z}_{\mathrm{bb}}^{\mathrm{r}} \mathbf{v}_{\mathrm{b}}^{\mathbf{r}}
\end{aligned}
$$

where; $\mathbf{Z}_{\mathbf{i j}}$ is the acoustic impedance between the DoFs $i$ and $j$, the subscripts $a$ and $b$ represent the coupling interface and remote receiver DoFs, respectively, and the superscripts $s$ and $r$ represent the source and receiver sub-systems, respectively. All variables are represented in the frequency domain, with explicit notation omitted for clarity.

The coupling of source and receiver sub-systems is achieved through the application of the continuity and compatibility conditions;

$$
\mathbf{p}_{\mathbf{a}}^{\mathbf{s}}=\mathbf{p}_{\mathbf{a}}^{\mathbf{r}} \text {, }
$$


and

$$
\mathbf{v}_{\mathbf{a}}^{\mathbf{s}}=\mathbf{v}_{\mathbf{a}}^{\mathbf{r}} \text {. }
$$

An additional third boundary condition is introduced here,

$$
\mathbf{p}_{\mathbf{b}}^{\mathbf{r}}=\mathbf{0}
$$

stating that no external pressures are applied at the remote DoF $b$.

Together, Eqs. (A.1)-(A.7) describe the dynamic behaviour of the coupled source-receiver assembly, whilst subjected to externally applied pressures at the internal source DoFs, $e$. This coupled assembly will be referred to hereafter as the General Case, for reasons that will shortly become clear.

Having defined the coupled assembly, our aim is now to find an equivalent excitation that, when applied to the source-receiver interface, $a$, in the absence of $\mathbf{p}_{\mathbf{e}}^{\mathbf{s}}$ (i.e. whilst the source is inactive), generates a response field in the receiver sub-system that is identical to the General Case. This will be achieved by considering the solution to the General Case as the sum of two simpler problems [14]. Following the formulation of Bobrovnitskii, these will be referred to as Auxiliary Case 1 and 2. The velocity response of the General Case is therefore given by,

$$
\begin{aligned}
& \mathbf{v}_{\mathrm{e}}^{\mathbf{s}}=\mathbf{v}_{\mathbf{e}_{1}}^{\mathbf{s}}+\mathbf{v}_{\mathrm{e}_{2}}^{\mathrm{s}} \\
& \mathbf{v}_{\mathbf{a}}^{\mathbf{s}}=\mathbf{v}_{\mathbf{a}_{1}}^{\mathbf{s}}+\mathbf{v}_{\mathbf{a}_{2}}^{\mathbf{s}} \\
& \mathbf{v}_{\mathbf{a}}^{\mathbf{r}}=\mathbf{v}_{\mathbf{a}_{1}}^{\mathbf{r}}+\mathbf{v}_{\mathbf{a}_{2}}^{\mathbf{r}} \\
& \mathbf{v}_{\mathbf{b}}^{\mathbf{r}}=\mathbf{v}_{\mathbf{b}_{1}}^{\mathbf{r}}+\mathbf{v}_{\mathbf{b}_{2}}^{\mathbf{r}}
\end{aligned}
$$

where subscripts 1 and 2 refer to the auxiliary case.

In what follows it will be shown that by applying a particular set of boundary conditions to Auxiliary Case 1, and solving for the boundary conditions of Auxiliary Case 2 (such that their sum returns the General Case), an equivalent kinematic excitation presents itself.

\section{Appendix A.2. Auxiliary Case 1}

With reference to Eqs. (A.8)-(A.11), the pressure-velocity relations of Auxillary Case 1 are given by,

$$
\begin{aligned}
& \mathbf{p}_{\mathrm{e} 1}^{\mathrm{s}}=\mathbf{Z}_{\mathrm{ea}}^{\mathrm{s}} \mathbf{v}_{\mathrm{a} 1}^{\mathrm{s}}+\mathbf{Z}_{\mathrm{ee}}^{\mathrm{s}} \mathbf{v}_{\mathrm{e} 1}^{\mathrm{s}} \\
& \mathbf{p}_{\mathrm{a} 1}^{\mathrm{s}}=\mathbf{Z}_{\mathrm{aa}}^{\mathrm{s}} \mathbf{v}_{\mathrm{a} 1}^{\mathrm{s}}+\mathbf{Z}_{\mathrm{ae}}^{\mathrm{s}} \mathbf{v}_{\mathrm{e} 1}^{\mathrm{s}} \\
& \mathbf{p}_{\mathrm{a} 1}^{\mathrm{r}}=\mathbf{Z}_{\mathrm{aa}}^{\mathrm{r}} \mathbf{v}_{\mathrm{a} 1}^{\mathrm{r}}+\mathbf{Z}_{\mathrm{ab}}^{\mathrm{r}} \mathbf{v}_{\mathrm{b} 1}^{\mathrm{r}} \\
& \mathbf{p}_{\mathrm{b} 1}^{\mathrm{r}}=\mathbf{Z}_{\mathrm{ba}}^{\mathrm{r}} \mathbf{v}_{\mathrm{a} 1}^{\mathrm{r}}+\mathbf{Z}_{\mathrm{bb}}^{\mathrm{r}} \mathbf{v}_{\mathrm{b} 1}^{\mathrm{r}} .
\end{aligned}
$$

In Auxiliary Case 1 we are free to choose an arbitrary set of boundary conditions. The boundary conditions of Auxiliary Case 2 will be derived with reference to those chosen here.

With the aim of independently characterising the source sub-system, the boundary conditions are chosen such that the source and receiver sub-systems are uncoupled (i.e. a pressure release boundary), with the pressure source, $\mathbf{p}_{\mathbf{e}}^{\mathbf{s}}$, still active.

$$
\begin{gathered}
\mathbf{p}_{\mathrm{e}_{1}}^{\mathrm{s}}=\mathbf{p}_{\mathrm{e}}^{\mathrm{s}} \\
\mathbf{p}_{\mathrm{a}_{1}}^{\mathrm{s}}=\mathbf{0} \\
\mathbf{p}_{\mathrm{a}_{1}}^{\mathrm{r}}=\mathbf{0} \\
\mathbf{p}_{\mathrm{b}_{1}}^{\mathrm{r}}=\mathbf{0}
\end{gathered}
$$

The above boundary conditions represent the uncoupled source and receiver sub-systems, as illustrated in figure A.15. Substituting these boundary conditions into Eqs. (A.12)-(A.15) yields,

$$
\mathbf{p}_{\mathrm{e}_{1}}^{\mathrm{s}}=\mathbf{Z}_{\mathrm{ee}^{\mathrm{s}}}^{\mathrm{s}} \mathbf{v}_{\mathrm{e}_{1}}^{\mathrm{s}}+\mathbf{Z}_{\mathrm{ea}^{\mathrm{s}} \mathbf{v}_{\mathbf{a}_{1}}^{\mathrm{s}}}^{\mathrm{s}}
$$




$$
\begin{gathered}
\mathbf{0}=\mathbf{Z}_{\mathbf{a e}^{\mathrm{a}} \mathbf{v}_{\mathbf{e}_{1}}^{\mathrm{s}}+\mathbf{Z}_{\mathbf{a a}^{2}}^{\mathrm{s}} \mathbf{v}_{\mathbf{a}_{1}}^{\mathrm{s}}} \mathbf{v}_{\mathbf{a}_{1}}^{\mathbf{r}}=\mathbf{0} \\
\mathbf{v}_{\mathbf{b}_{1}}^{\mathbf{r}}=\mathbf{0}
\end{gathered}
$$

where we note that, as expected, the velocity field of the uncoupled receiver sub-system is 0 .

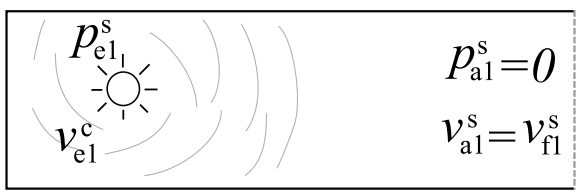

$\begin{array}{ll}p_{\mathrm{a} 1}^{\mathrm{r}}=0 & \text { 우 } \begin{array}{l}p_{\mathrm{b} 1}^{\mathrm{r}}=0 \\ \nu_{\mathrm{a} 1}^{\mathrm{r}}=0\end{array} \\ \end{array}$

Figure A.15: Auxiliary Case 1

The application of a pressure release boundary condition may be considered analogous to the free suspension of a vibration source. As such, we will refer to the interface velocity of the uncoupled source sub-system as an acoustic 'free velocity' and denote it, $\mathbf{v}_{\mathbf{a}_{1}}^{\mathbf{s}}=\mathbf{v}_{\mathbf{f}}^{\mathbf{s}}$. As in the structural case, the acoustic free velocity is an intrinsic property of the source sub-system, and independent of the (now uncoupled) receiver sub-system.

\section{Appendix A.3. Auxiliary Case 2}

The boundary conditions of Auxiliary Case 2 must now be determined such that the sum of Auxiliary Case 1 and 2 yields the General Case. It will be shown that the resultant boundary conditions describe the sought after equivalent kinematic excitation.

Substituting Eqs. (A.8) and (A.9) (the decomposed velocity response at DoFs $e$ and $a$ ) into Eq. (A.1) of the General Case yields,

$$
\mathbf{p}_{\mathrm{e}}^{\mathrm{s}}=\mathbf{Z}_{\mathrm{ea}}^{\mathrm{s}}\left(\mathbf{v}_{\mathbf{a}_{1}}^{\mathrm{s}}+\mathbf{v}_{\mathbf{a}_{2}}^{\mathrm{s}}\right)+\mathbf{Z}_{\mathrm{ee}}^{\mathrm{s}}\left(\mathbf{v}_{\mathbf{e}_{1}}^{\mathrm{s}}+\mathbf{v}_{\mathrm{e}_{2}}^{\mathrm{s}}\right)=\mathbf{p}_{\mathrm{e}_{1}}^{\mathrm{s}}+\mathbf{p}_{\mathrm{e}_{2}}^{\mathrm{s}} .
$$

Noting Eqs. (A.16) and (A.20) (the definitions resulting from our choice of boundary conditions in Auxiliary Case 1), it can be seen that for the above to be satisfied, $\mathbf{p}_{\mathbf{e}_{2}}^{\mathbf{s}}=\mathbf{Z}_{\mathbf{e e}}^{\mathbf{s}} \mathbf{v}_{\mathbf{e}_{2}}^{\mathbf{s}}+\mathbf{Z}_{\mathbf{e a}_{\mathbf{a}}}^{\mathbf{s}} \mathbf{v}_{\mathbf{a}_{2}}^{\mathbf{s}}=\mathbf{0}$, must hold. Auxiliary Case 2 therefore represents a system in which the pressure source, $\mathbf{p}_{\mathbf{e}_{2}}^{\mathbf{s}}$, is inactive.

Enforcement of $\mathbf{p}_{\mathbf{e}_{2}}^{\mathbf{s}}=\mathbf{0}$ (whilst noting that $\mathbf{p}_{\mathbf{b}}^{\mathbf{r}}=\mathbf{0}$ ) yields the pressure-velocity relations of Auxiliary Case 2,

$$
\begin{aligned}
& \mathbf{0}=\mathbf{Z}_{\mathrm{ea}^{2}}^{\mathrm{s}} \mathbf{v}_{\mathbf{a}_{2}}^{\mathrm{s}}+\mathbf{Z}_{\mathrm{ee}^{\mathrm{e}}}^{\mathrm{s}} \mathbf{v}_{\mathrm{e}_{2}}^{\mathrm{s}} \\
& \mathbf{p}_{\mathrm{a}_{2}}^{\mathrm{s}}=\mathbf{Z}_{\mathrm{aa}}^{\mathrm{s}} \mathbf{v}_{\mathbf{a}_{2}}^{\mathrm{s}}+\mathbf{Z}_{\mathrm{ae}}^{\mathrm{s}} \mathbf{v}_{\mathrm{e}_{2}}^{\mathrm{s}} \\
& \mathbf{p}_{\mathrm{a}_{2}}^{\mathrm{r}}=\mathbf{Z}_{\mathrm{aa}}^{\mathrm{r}} \mathbf{v}_{\mathrm{a}_{2}}^{\mathrm{r}}+\mathbf{Z}_{\mathrm{ab}}^{\mathrm{r}} \mathbf{v}_{\mathrm{b}_{2}}^{\mathrm{r}} \\
& \mathbf{0}=\mathbf{Z}_{\mathbf{b a}^{2}}^{\mathbf{r}} \mathbf{v}_{\mathbf{a}_{2}}^{\mathbf{r}}+\mathbf{Z}_{\mathbf{b b}_{\mathbf{b}}}^{\mathbf{r}} \mathbf{v}_{\mathbf{b}_{2}}^{\mathbf{r}} \text {. }
\end{aligned}
$$

Following the substitution of Eq. (A.2) and (A.3) into Eq. (A.5), whilst taking account of the auxiliary definitions, $\mathbf{v}_{\mathbf{a}}^{\mathbf{s}}=\mathbf{v}_{\mathbf{a}_{1}}^{\mathbf{s}}+\mathbf{v}_{\mathbf{a}_{2}}^{\mathbf{s}}$ and $\mathbf{v}_{\mathbf{a}}^{\mathbf{r}}=\mathbf{v}_{\mathbf{a}_{1}}^{\mathbf{r}}+\mathbf{v}_{\mathbf{a}_{2}}^{\mathbf{r}}$, the equilibrium condition of the General Case, $\mathbf{p}_{\mathbf{a}}^{\mathbf{s}}=\mathbf{p}_{\mathbf{a}}^{\mathbf{r}}$, is given by,

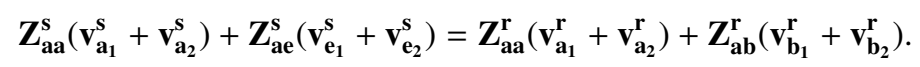

Expanding the above whilst noting the definitions resulting from Auxiliary Case 1 [Eqs. (A.21)-(A.23)],

$$
\mathbf{Z}_{\mathbf{a e}}^{\mathrm{s}} \mathbf{v}_{\mathbf{e}_{2}}^{\mathrm{s}}+\mathbf{Z}_{\mathbf{a a}}^{\mathrm{s}} \mathbf{v}_{\mathbf{a}_{2}}^{\mathrm{s}}=\mathbf{Z}_{\mathbf{a a}_{2}}^{\mathrm{r}} \mathbf{v}_{\mathbf{a}_{2}}^{\mathrm{r}}+\mathbf{Z}_{\mathbf{a b}^{\mathrm{b}}}^{\mathrm{r}} \mathbf{v}_{\mathbf{b}_{2}}^{\mathrm{r}}
$$

we arrive at the equilibrium boundary condition of Auxiliary Case 2,

$$
\mathbf{p}_{\mathrm{a}_{2}}^{\mathrm{s}}=\mathbf{p}_{\mathrm{a}_{2}}^{\mathbf{r}} .
$$

Substituting the auxiliary definitions into the compatibility condition of the General Case [Eq. (A.6)] yields,

$$
\mathbf{v}_{\mathbf{a}}^{\mathbf{s}}=\mathbf{v}_{\mathbf{a}}^{\mathbf{r}} \longrightarrow \mathbf{v}_{\mathbf{a}_{1}}^{\mathbf{s}}+\mathbf{v}_{\mathbf{a}_{2}}^{\mathbf{s}}=\mathbf{v}_{\mathbf{a}_{1}}^{\mathbf{r}}+\mathbf{v}_{\mathbf{a}_{2}}^{\mathbf{r}} .
$$


Noting that from Auxiliary Case 1, $\mathbf{v}_{\mathbf{a}_{1}}^{\mathbf{s}}=\mathbf{v}_{\mathbf{f}}^{\mathbf{s}}$ and $\mathbf{v}_{\mathbf{a}_{1}}^{\mathbf{r}}=\mathbf{0}$, the compatibility condition of Auxiliary Case 2 is found,

$$
\mathbf{v}_{\mathbf{a}_{2}}^{\mathbf{r}}-\mathbf{v}_{\mathbf{a}_{2}}^{\mathbf{s}}=\mathbf{v}_{\mathbf{f}}^{\mathbf{s}} \text {. }
$$

Together, Eqs. (A.31) and (A.33), describe the boundary conditions required by Auxiliary Case 2, such that the sum of Auxiliary Case 1 and 2 yields the General Case. This assembly is illustrated in figure A.16.

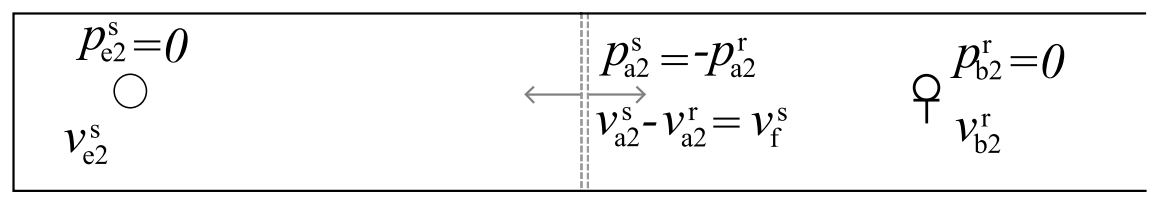

Figure A.16: Auxiliary Case 2

The above states that the response field in the receiver of a coupled assembly may be replicated identically through a kinematic excitation at the coupling interface that is proportional to the free velocity of the uncoupled source subsystem. The reader may be convinced of this by considering the following. In Auxiliary Case 1 the source and receiver sub-systems are uncoupled and the receiver's response field is 0. In Auxiliary Case 2 the source and receiver sub-systems are coupled via a load that generates a kinematic response at the coupling interface. Since the sum of the two auxiliary cases must be equal to the general coupled case, this kinematic excitation must reproduce an identical response field in the receiver as per the General Case. This suggests that there exists a kinematic quantity that may be determined from a coupled assembly (i.e. Auxiliary Case 2), that is directly proportional to the source subsystem's free velocity. It is this notion that forms the theoretical basis of the proposed independent characterisation procedure. Confidence in the above is further gained from knowledge that a similar equivalent field representation has successfully been used to independently characterise vibration sources [30].

\section{Appendix B. Post Processing for Auralisation}

A requirement of the proposed inverse approach is the existence of a reliable phase relationship between the elements of the operational pressure vector, $\mathbf{p}=\left[p_{1}, p_{2}, \cdots, p_{n}\right]^{T}$. Classically, in both acoustic and vibro-acoustic applications, this type of operational vector is determined from time averaged auto and cross-spectra, where the crossspectrum angle between each signal and a reference is assigned as the phase of the appropriate auto-spectrum [31], as per Eq. (B.1),

$$
\left[\begin{array}{c}
\hat{p}_{1} \\
\hat{p}_{2} \\
\vdots \\
\hat{p}_{n}
\end{array}\right]=\left[\begin{array}{c}
\sqrt{<S_{11}>} \\
\sqrt{<S_{22}>} \\
\vdots \\
\sqrt{<S_{n n}>}
\end{array}\right] \odot\left[\begin{array}{c}
e^{i 0} \\
e^{i<<S_{12}>} \\
\vdots \\
e^{i<<S_{1 n}>}
\end{array}\right]
$$

where, $\odot$ and $<>$ represent the Hadamard (element-wise) product and time averaged quantities, respectively.

Although a well established method, this cross-spectrum phase approach not only relies on a constant phase relationship, but assumes a steady state source behaviour. Although a fair assumption in most cases, with the aim of producing realistic auralisations in mind, such an approach is unlikely to be suitable. The human ear is particularly sensitive in detecting small temporal variations in auralised signals. As such, it is proposed that the time structure of the source be retained, and any averaging processes used only in the graphical presentation of data.

As an alternative it is suggested that sequential Fourier spectra be used. This approach makes no assumptions regarding the operational behaviour of the source and retains its time structure through a time varying source quantity. The sequential Fourier spectra approach may be formulated as per Eq. (B.2), where $\mathcal{F}\left\{P_{n}\left(\Delta t_{m}\right)\right\}$ represents the Fourier transform of the $n$th time domain pressure signal, $P$, over the $m$ th time window, $\Delta t$.

$$
\left[\begin{array}{c}
p_{1} \\
p_{2} \\
\vdots \\
p_{n}
\end{array}\right]_{m}=\left[\begin{array}{c}
\mathcal{F}\left\{P_{1}\left(\Delta t_{m}\right)\right\} \\
\mathcal{F}\left\{P_{2}\left(\Delta t_{m}\right)\right\} \\
\vdots \\
\mathcal{F}\left\{P_{n}\left(\Delta t_{m}\right)\right\}
\end{array}\right]
$$


For a given time window the $n$ Fourier spectra are phase referenced to the beginning of that window and a meaningful phase relationship is established.

The operational pressure vector $\mathbf{p}=\left[p_{1}, p_{2}, \cdots, p_{n}\right]^{T}$ is thus replaced by $\mathbf{p}_{m}=\left[p_{1}, p_{2}, \ldots p_{n}\right]_{m}^{T}$ and the inverse procedure repeated for $m \rightarrow M$ time windows, where $M$ is the total number of time windows used. The resulting time varying volume velocities (of which there will be $M$ ) may be used in the prediction of an operational response, the results of which may be inverse Fourier transformed and recombined to yield an auralisation of a 'virtual' assembly.

\section{References}

[1] A.T. Moorhouse. Virtual acoustic prototypes: Listening to machines that don't exist. In Proceeedings of Acoustics 2005, Busselton, Western Australia, pages 11-22, 2005.

[2] International Organization for Standardization. BE EN ISO 5136 : Acoustics - Determination of sound power radiated into a duct by fans and other air-moving devices - In-duct method, 2009.

[3] P.K. Baade. Effects of acoustic loading on axial flow fan noise generation. Noise Control Engineering Journal, 8(1):5-15, 1976.

[4] W. Neise and F. Arnold. On sound power determination in flow ducts. Journal of Sound and Vibration, 244(3):481-503, 2001.

[5] K.A. Edge and D.N. Johnston. The 'Secondary Source' Method for the Measurement of Pump Pressure Ripple Characteristics Part 1: Description of Method. Proceedings of the Institution of Mechanical Engineers, Part A: Journal of Power and Energy, 204(1):33-40, 1990.

[6] International Organization for Standardization. ISO 10767-1:2015 Hydraulic fluid power - Determination of pressure ripple levels generated in systems and components. Part 1: Method for determining source flow ripple and source impedance of pumps, 2015.

[7] J. Lavrentjev, M. Abom, and H. Boden. A measurement method for determining the source data of acoustic two-port sources. Journal of Sound and Vibration, 183(3):517-531, 1995.

[8] M. Abom. Measurement of the scattering-matrix of acoustical two-ports. Mechanical Systems and Signal Processing, 5(2):89-104, 1991.

[9] M. Abom and H. Boden. A note on the aeroacoustic source character of in-duct axial fans. Journal of Sound and Vibration, 186(4):589-598, 1995.

[10] J. Lavrentjev and M. Abom. Characterisation of fluid machines as acoustic multi-port sources. Journal of Sound and Vibration, 197(1):1-16, 1996.

[11] A. Holmberg, M. Abom, and H. Boden. Accurate experimental two-port analysis of flow generated sound. Journal of Sound and Vibration, 330(26):6336-6354, 2011.

[12] L. Mongeau, De E. Thompson, and De K. McLaughlin. A method for characterizing aerodynamic sound sources in turbomachines. Journal of Sound and Vibration, 181:369-389, 1995.

[13] A.T. Moorhouse, A.S. Elliot, and Y.H. Heo. Intrinsic characterisation of structure-borne sound sources and isolators from in-situ measurements. In Proceedings of Meetings on Acoustics, Montreal, Canada, volume 19, 2013.

[14] Y.I. Bobrovnitskii. A theorem on the representation of the field of forced vibrations of a composite elastic system. Acoustical Physics, 47(5):586-589, 2001.

[15] Y.I. Bobrovnitskii and G. Pavic. Modelling and characterization of airborne noise sources. Journal of Sound and Vibration, 261(3):527-555, mar 2003.

[16] R. Penrose and J.A. Todd. A generalized inverse for matrices. Mathematical Proceedings of the Cambridge Philosophical Society, 51(03):406, 1955.

[17] T. Salava. Acoustic load and transfer functions in rooms at low frequencies. Journal of the Audio Engineering Society, 36(10):763-775, 1988.

[18] N. Moller, S. Gade, J. Hald, and D.K. Nærum. The use of volume velocity source in transfer measurements. Technical report, SAE Technical Paper, 2004.

[19] S. Gade, N. Møller, J. Hald, and L. Alkestrup. The use of Volume Velocity Source in Transfer Measurements. Internoise2004. Proceedings of 33th International Congress on Noise Control Engineering, V:1-8, 2004.

[20] Siemens LMS. Mid/High-Frequency Volume Soure, 2015.

[21] Brüel \& Kjær. OmniSource with 4299 Volume Velocity Adaptor - Type 4295, 2015.

[22] P.M. Morse and K.U. Ingard. Theoretical Acoustics. Princeton University Press, New Jersey, 1st edition, 1968.

[23] L.J. Eriksson. Higher order mode effects in circular ducts and expansion chambers. The Journal of the Acoustical Society of America, 68(2):545, 1980.

[24] T.J. Newman. Towards a silent fan: an investigation of low-speed fan aeroacoustics. PhD thesis, University of Cambridge, 2014.

[25] A.N. Thite and D.J. Thompson. The quantification of structure-borne transmission paths by inverse methods. Part 1: Improved singular value rejection methods. Journal of Sound and Vibration, 264(2):411-431, 2003.

[26] A.N. Thite and D.J. Thompson. The quantification of structure-borne transmission paths by inverse methods. Part 2: Use of regularization techniques. Journal of Sound and Vibration, 264(2):433-451, 2003.

[27] J. Woodhouse. An introduction to statistical energy analysis of structural vibration. Applied Acoustics, 14(6):455-469, 1981.

[28] B.M. Gibbs, R. Cookson, and N. Qi. Vibration activity and mobility of structure-borne sound sources by a reception plate method. The Journal of the Acoustical Society of America, 123(6):4199-209, 2008.

[29] G. Banwell, H. Hopper, A.T. Moorhouse, A.S. Elliott, and J.W.R. Meggitt. Methods for auralising sound with tonal components. In Proceedings of the International Congress on Sound and Vibration, Florence, Italy, number 22, 2015.

[30] A.T. Moorhouse, T.A. Evans, and A.S. Elliott. Some relationships for coupled structures and their application to measurement of structural dynamic properties in situ. Mechanical Systems and Signal Processing, 25(5):1574-1584, jul 2011.

[31] M.H.A. Janssens, J.W. Verheij, and D.J. Thompson. The use of an equivalent forces method for the experimental quantification of structural sound transmission in ships. Journal of Sound and Vibration, 226(2):305-328, sep 1999. 